\title{
SURFACE MODIFICATION OF COTTON FABRICS FOR SUBLIMATION PRINTING
}

\author{
Jadwiga Bemska', Joanna Szkudlarek ${ }^{2}$ \\ ${ }^{1}$ Lodz University of Technology, Faculty of Material Technologies and Textile Design, \\ Department of Man-Made Fibres, Żeromskiego St 116, Łódź 90-924, Poland \\ ${ }^{2}$ Lodz University of Technology, Faculty of Material Technologies and Textile Design, \\ Institute of Architecture of Textiles, Żeromskiego St 116, Łódź 90-924, Poland \\ E-mail: jbemska@p.lodz.pl, jmszkudlarek@poczta.onet.pl
}

\begin{abstract}
:
The use of sublimation thermal transfer printing onto cotton is not widely practiced because of the lack of disperse dyes affinity for cellulose fibres. The studies were performed onto polymer application on cotton fibres in order to achieve the ability to absorption of sublimating dyes and thus the possibility of making printouts onto cotton. The following parameters were evaluated: whiteness degree after impregnation, the relative strength of colours of the obtained printouts, test washing fastness, and dry and wet rubbing fastness.
\end{abstract}

\section{Keywords:}

Transfer printing, digital printing, sublimation printing, disperse dyes, cotton, surface modification of cotton, pattern

\section{Introduction}

The technology of sublimation transfer printing is a very attractive method of decorating fabrics of polyester [1-5]. It enables to obtain a multi-coloured prints of digital quality, i.e. extreme tonal effects and fine lines. Typically, the prepared pattern is printed on special paper by ink-jet printer containing disperse dyes capable of sublimation, and then the dried pattern is heat transferred to the fabric. Sublimation phenomenon is possible due to the fact that disperse dyes contained in the inks of printer are relatively small, non-polar molecules [3].

Due to such property, the dyes in the process of heating under the press are able to go into steam and diffuse into polyester fibres. The disperse dyes have an affinity for hydrophobic fibre first of all for polyester.

One major advantage of transfer printing is that the resulting printouts do not require additional curing or wet finishing, cleansing and drying after them, as there are no chemicals and very little surface dye to remove from the fabric.
The other advantage of this method is the possibility to achieve transferred prints that are resistant to light, rubbing and wet operations. Wash fastness is very high [1-3].

The aim of this research is such modification of cotton surface, by the application of the synthetic polymer, in order to give it the ability to absorb disperse dyes. This ability allows to apply disperse dyes (capable of sublimation) by thermal transfer from paper to cotton. In the case of non-modified cellulose fibres, it is not possible to obtain satisfactory colour effects and resistance because the disperse dyes have no affinity for cellulosic material.

\section{Materials and methods}

\section{Materials}

Agents based on polymeric resins were applied onto knitted cotton fabrics of $140 \mathrm{~g} / \mathrm{m}$ commonly used for T-shirt confectioning. Table 1 presents the list of agents with technical description.

Table 1. List of the Modification Agents.

\begin{tabular}{|c|c|c|c|}
\hline Lp & Name/producer & Chemical structure & Functional properties [7] \\
\hline 1 & FIXATIVE ABN [TEXCHEM] & Self-crosslinking butadiene-acrylonitrile resin & Binder for printing \\
\hline 3 & POLAPPRET AC-S[ZSM] & Acrylic copolymer & Filling agent with flexible grip \\
\hline 2 & ACRILEM [ICAP-SIRA] & Dispersion of styrene-acrylic copolymer & Binder for printing \\
\hline 4 & POLAPPRET PU-S [ZSM] & Polyurethane dispersion & Filling agent with soft, flexible grip \\
\hline 5 & UNIGUARD Y [TEXCHEM] & Blocked isocyanate & Crosslinker \\
\hline 6 & UTRWALACZ W [TEXCHEM] & Derivative of melamine & Softener \\
\hline 7 & ZMIĘKCZACZ ICAP SIRA & Wax dispersion & \\
\hline
\end{tabular}


The listed agents indicate different effectiveness according to thermal transfer and different fastness to washing. Some of them cause slightly yellowing of the white knitted cotton fabric. For the optimisation research commercial products POLAPPRET AC-S (A) and POLAPPRET PU-S (P) were selected for the reasons of the low yellowing of the modified surface and satisfactory colour results after the washing process. These agents were applied with isocyanate-based crosslinking agents and the modified melamine: UNIGARD $Y$ (Y) or Utrwalacz W (W). The mentioned agents were used in an amount of $20 \%$ in the ratio of used resin weight. The prepared compositions are marked as AY, AW, PY, PW.

These compositions were applied in aqueous solutions at various concentrations in the range of $20-100 \%$ of commercial products in padding technology, squeeze degree of $30 \%$. The padded samples were dried at $90^{\circ} \mathrm{C}$ for 3 minutes, and then cured at $150^{\circ} \mathrm{C}$ for 3 minutes. Sublimation printing papers were printed on Epson Stylus Photo 1400, the basic CMYK colours as rectangles of colour parameters of $100 \%$ for each colour component.

The transfer was carried out on the press Blue PRESSLine f-my Schulz at $180^{\circ} \mathrm{C}$ for 50 seconds. For comparison under the same conditions, transfer was performed on the knitted PET fabric.

\section{Evaluation methodology of research results}

The colour parameters of and whiteness of the printouts were assessed on spectral reflectometer Macbeth Colour Eye 3000 by the determination of the remission value corresponding to the light wavelengths in the range $400-700 \mathrm{~nm}$.

The background whiteness of the printout was estimated on the basis of the remission of the modified fabric using the Stephenson formula:

$$
W=2 B-R
$$

where $W$ is the whiteness degree, $B(\%)$ the remission of blue light, corresponding to the radiation of the wavelength $\lambda=460 \mathrm{~nm}, R(\%)$ the remission of red light, corresponding to the radiation of wavelength $\lambda=615 \mathrm{~nm}$.

For the individual chromatical colour of the printouts, the values of the relative strength of colour ( $K / S$ value) were calculated in accordance with Kubelka Munk's equation:

$$
\frac{K}{S}=\frac{(1-R)^{2}}{2 R}
$$

where $R$ is the remission value corresponding to the wavelength of light, for which remission is minimal, $K$ the absorption coefficient and $S$ the scattering coefficient.

Evaluation of washing fastness was based on the standards $\mathrm{PN}-\mathrm{ISO}$ 105-C06. Test washing process was performed with the dyeing laboratory apparatus (Labomat-Mathis) at $40^{\circ} \mathrm{C}$.

Evaluation of rubbing fastness was based on the European standard: textiles - tests for colour fastness - Vol. X12: Colour fastness to rubbing PN-EN ISO 105-X12. The degree of whiteness contamination of dry and wet rubbing fabric was assessed.

For test, samples were specified:

1. The whiteness degree $(W)$ of modified knitted fabric, which represents the white background of printout, was measured before and after the thermal transfer (Figure 1).

2. The relative strength of the colour ( $K / S$ value) for each primary colour of printouts, before and after washing (Figure 3 ).

3. The printouts fastness to dry and wet rubbing and washing for optimal composition of agents (Table 2).

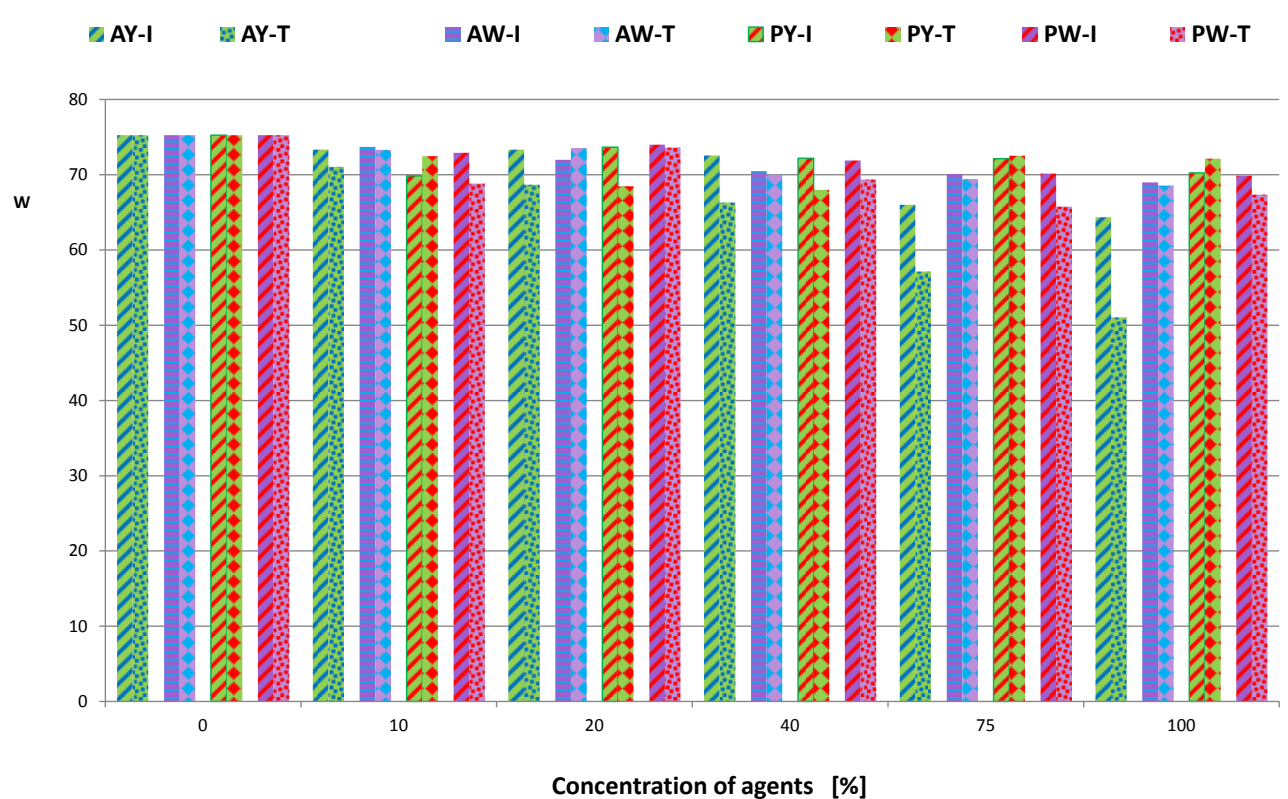

Figure 1. The values of the whiteness degree of the fabric impregnated and cured before $(I)$ and after the transfer $(T)$. 


\section{Results and discussion}

Analysis of the whiteness degree of the modified fabric leads to the conclusion that the white background does not change significantly during subsequent thermal processing, although heat treatment of resin agents, both during curing (after impregnation) and the transfer printing carries such risk (Figure 1).

Figure 2 shows that with the growth of the concentration of applied agents, the whiteness degree of printing background slightly decreases especially in the case of POLAPPRET AC-S (A).

The washing process reliably assesses the usefulness of the used composition for impregnation.

During washing process, the removal of dyes from printing surface occurs. Migration of the dye to the bath cures the risk of reabsorption onto the treated surface fibres. Such a mechanism can explain the phenomenon of white background contamination of the printout, which is intensified at low concentration of impregnating agents.
In case of low concentration of modifiers, more particles of dye migrate into the wash liquor, from where they move to the surface of the modified print background. Background staining is observed at concentrations below $60 \%$.

Evaluation of samples background points out that the least dirty background has the fabric modified with agent - POLAPPRET PU-S with the addition of UNIGUARD Y (PY). Simultaneously, the degree of whiteness of the fabrics impregnated with this agent measured before the washing process is the highest (Figure 1).

The research shows that the relative strength of colour of printouts assessed by the determination of $\mathrm{K} / \mathrm{S}$, the ratio before and after washing, reaches the satisfactory level according to PES. Figure 3 shows the comparison of the intensity values of colour of printouts before and after washing (W).

From the analysis of the graphs, it appears that the most effective agent is POLAPPRET PU-S used in combination with UNIGUARD Y, and then POLAPPRET AC-S used in the compositions; but the quality of the crosslinking agents does not matter. Relatively, POLLAPRET PU-S in combination with Utrwalacz $\mathrm{W}$ has the lowest efficiency.

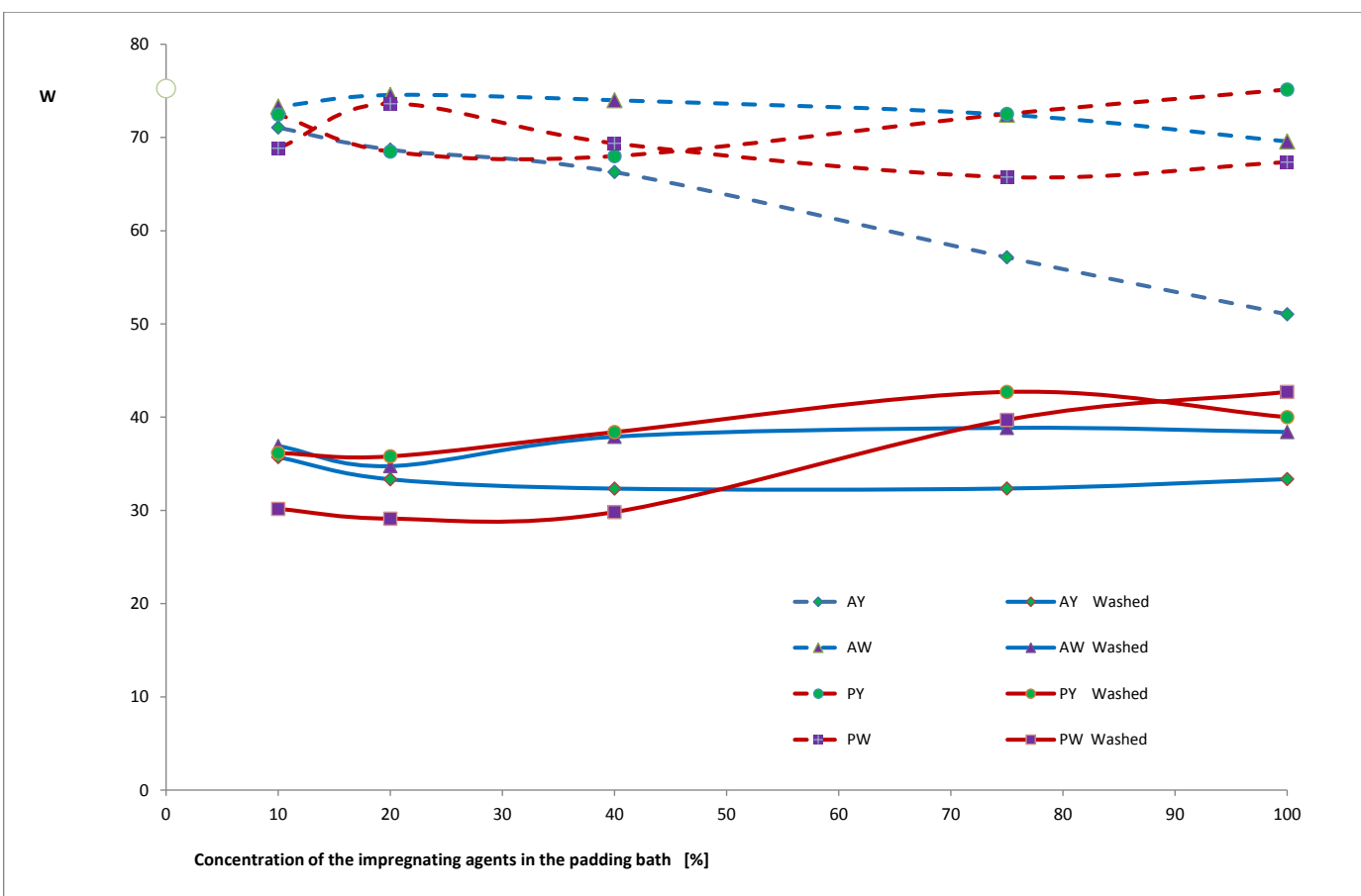

Figure 2. Influence of concentration of impregnating agents on whiteness degree of the print background before and after washing (washed).

Table 2. Washing Fastness and Dry and Wet Rubbing Fastness of Printouts for Optimal Composition of PY $75 \%$

\begin{tabular}{|c|c|c|c|c|c|}
\hline \multirow{2}{*}{ Colour } & \multicolumn{2}{|c|}{ Rubbing } & \multicolumn{2}{c|}{ Washing } \\
\cline { 2 - 6 } & $\begin{array}{c}\text { Staining degree } \\
\text { of dry fabric }\end{array}$ & $\begin{array}{c}\text { Staining degree } \\
\text { of wet fabric }\end{array}$ & $\begin{array}{c}\text { Degree of change } \\
\text { colour }\end{array}$ & \multicolumn{2}{c|}{ Staining degree of whiteness } \\
\hline C & 5 & 5 & 5 & 5 & CO \\
\hline M & 5 & $4 / 5$ & 5 & 5 & 5 \\
\hline Y & 5 & $4 / 5$ & 5 & 5 & 5 \\
\hline K & 5 & $4 / 5$ & 5 & 5 & 5 \\
\hline
\end{tabular}



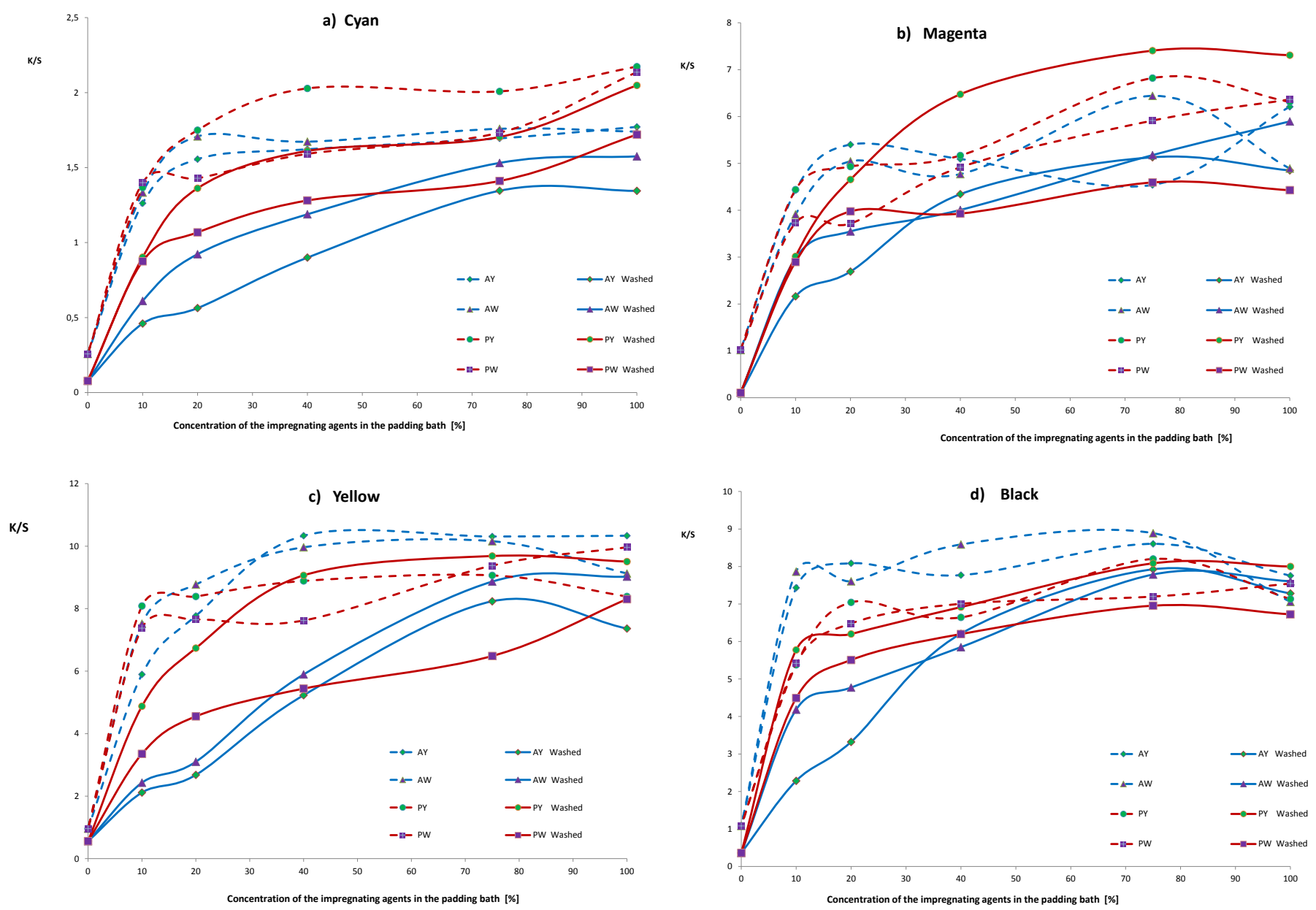

Figure 3. The influence of concentration of impregnating agents on the colour of printouts before and after washing (Washed).

We find the most effective composition PY (POLAPPRET PU-S with the addition of UNIGUARD Y) at a concentration of $75 \%$, which is used for further studies, such as assessment of resistance to wet and dry rubbing and washing. The washing fastness is determined with cotton and polyester companion fabric (Table 2).

Resistance to both rubbing and washing is very good.

The whiteness degree of fabrics representing white print background, after the impregnation of the agents POLAPPRET PU-S and POLAPPRET AC-S, does not change significantly despite thermal treatment, both during curing and transfer printing. Used combinations demonstrate significant efficacy. For aqueous solutions of impregnating agents at concentrations above $60 \%$, the highest intensity of each primary colour CMYK can be achieved; the resistance to washing and rubbing (wet and dry) is at very good level. Danger of dirty background can be reduced by higher concentrations of modifying agents.

\section{Conclusions}

The results of performed studies indicate that there is a possibility of the application of disperse dyes on cotton products by sublimation printing method. As a result of modifying the surface of cotton fabric by POLAPPRET PU-S, we can improve the durability of printouts and simultaneously maintain colour quality at the expected level. It can open a new perspective for the digital transfer printing on cotton products.

\section{References}

[1] Miles, L.,: Textile Printing, Second Edition, Society of Dyers and Colourists 2003.

[2] Ingamells, W.,: Colour for Textiles, A User's Handbook, Society of Dyers and Colourists 1993.

[3] Lewin, M.,: Handbook of Fiber Chemistry, International Fiber Science and Technology CRC 2007.
[4] Rouette, H.,: Encyclopedia of Textile Finishing, Springer, 2001.

[5] Ujiie, H.,: Digital Printing of Textiles, Woodhead Publishing Limited and CRC Press LLC 2006.

[6] Teodorczyk , J.,: Technology and Equipment for Transfer Printing, Świat Druku 1/2005.

[7] Handouts of ZSM and TEXCHEM Companies. 